\title{
Lapurdum
}

Euskal ikerketen aldizkaria | Revue d'études basques |

Revista de estudios vascos | Basque studies review

2 | 1997

Numéro II

\section{Une confrérie originale au Moyen Âge : l'Armandat du pays de Labourd}

\section{Maïté Lafourcade}

\section{OpenEdition \\ Journals}

Édition électronique

URL : http://journals.openedition.org/lapurdum/1825

DOI : 10.4000/lapurdum.1825

ISSN : 1965-0655

\section{Éditeur}

IKER

Édition imprimée

Date de publication : 1 octobre 1997

Pagination : 293-301

ISBN : 2-84127-142-0

ISSN : $1273-3830$

Référence électronique

Maïté Lafourcade, « Une confrérie originale au Moyen Âge : I'Armandat du pays de Labourd »,

Lapurdum [En ligne], 2 | 1997, mis en ligne le 01 septembre 2010, consulté le 29 janvier 2020. URL:

http://journals.openedition.org/lapurdum/1825; DOI : 10.4000/lapurdum.1825 


\section{UNE CONFRÉRIE ORIGINALE AU MOYEN ÂGE : L'ARMANDAT DU PAYS DE LABOURD}

Si la vicomté de Labourd, fondée en $1023^{\prime}$ par Sanche III le Grand, roi de Navarre, fut, à la mort de ce grand roi, séparée du royaume de Navarre et incorporée en 1058, au duché d'Aquitaine, elle conserva néanmoins des liens très forts avec la Navarre ${ }^{2}$, au besoin pour résister au pouvoir des ducs d'Aquitaine. Ces liens se distendirent lorsqu'elle devint anglaise par le mariage, en 1152, de l'héritière du duché d'Aquitaine, Aliénor, avec Henri II Plantagenêt, roi d'Angleterre deux ans plus tard, à la mort de son père. Elle conserva toutefois le caractère basque de ses origines, étant beaucoup plus toumée vers le sud que vers le nord dont elle était séparée par les vastes landes marécageuses de Gascogne.

Après une révolte des seigneurs du sud-ouest, Richard Coeur de Lion, roi d'Angleterre et duc d'Aquitaine, vint, en 1177, mettre de l'ordre dans ses possessions d'Aquitaine. Vers 1190, il rendit une ordonnance, conservée aux archives municipales de Bayonne", "pour la répression des crimes et délits dans la ville et dans la vicomté de Bayonne" " il s'agit d' un véritable code de droit pénal, promulgué par le roi-duc avec l'assentiment des évêques de Dax et de Bayonne, du sénéchal de Gascogne, des seigneurs "cavers" s et du peuple, qui s'imposait à toute la vicomté. Le dernier vicomte de Labourd, disparu en $1193^{\circ}$, fut remplacé, à une date incertaine, par un bayle nommé par le roi et placé sous l'autorité du sénéchal de Guyenne, agent direct du roi d'Angleterre ; cet officier apparaît pour la première fois dans les textes en $1244^{7}$. Bayonne, qui participa au vaste mouvement de renouveau urbain du XII' siècle, repeuplée en partie de Gascons, fut alors séparée administrativement du Labourd.

Dès lors les Labourdins relevèrent directement, sans intermédiaire, du roi d'Angleterre - duc d'Aquitaine. L'enquête que fit faire Edouard II, après une période troublée, pour connaître ses droits sur la terre de Labourd, dont une copie de 1566 est conservée aux archives municipales de Bayonne", montre que "tora terra de Labourt tenetur a domino nostro Angliae rege duce Aquitaniae antiquitus immediate per nobiles et habitantes in terra predicta..." ". Il n'y avait donc pas d'intermédiaire entre le peuple labourdin et le roi d'Angleterre. pas de hiérarchie seigneuriale. Tous les habitants. nobles et non nobles, placés sur un pied d'égalité vis à vis de leur suzerain, lui devaient également l'hommage et les services vassaliques qui sont précisés par certains témoins: “... tenentur dictae gentes sequi dominum regem cum armis et facere host et cavalgada propriis suis expensis usque ad portum de Caulas et exinde expensis regis ubi sibi placuerit in ducatu Aquitaniae ad certum tempus, videlicet quadraginta dies..." "Les témoins interrogés signalent cependant l'existence de soixante à soixante-dix "homines regis ou proprios", appelés ailleurs "homines ligios, homines legei vel curiae" ", qui ne se distinguent des autres habitants que par 
l'albergade qu'ils doivent au roi et pour laquelle ils payent 2 sous 6 deniers morlans ${ }^{12}$ par an, et ils dénoncent les usurpations de droits seigneuriaux commises par quatre seigneurs : Guillaume Arnaud de Sault, le seigneur d'Espelette, celui de Pagandure et celui de Lahet qui exercent la Basse justice sur des terres qu'ils ont abusivement peuplées ${ }^{\text {t?. }}$.

Dès qu'il y avait une tentative d'usurpation de droits seigneuriaux en Labourd ou de concession par le roi de droits sur la terre basque, les Labourdins réagissaient vivement. Les exemples abondent ${ }^{14}$. Ainsi, en juillet 1341, les habitants du pays de Labourd adressèrent au roi Edouard III une plainte contre Amaud de Durefort qui avait "surpris" du roi la concession de terres en Labourd et ils obtinrent la révocation des concessions faites ${ }^{15}$.

La féodalité, à part quelques exceptions, ne put donc s'imposer en terre labourdine. C'est le bayle, représentant du roi, qui exerçait les droits de justice.

Mais il était, au XIVc siècle, confronté à de graves troubles, notamment occasionnés par les raids de seigneurs de pays voisins, accompagnés de bandes armées, qui, leur forfait accompli, s'en retournaient, impunis, dans leur territoire. Les archives municipales de Bayonne en conservent des témoignages, tel le raid, le 29 août 1400 , de Fernand Périt de Ayala qui pénétra en Labourd, avec 5000 combattants, s'empara de l'église de Saint-Jean-de-Luz et d'une partie du pays ${ }^{16}$.

Un pays montagneux et peu peuplé comme le Labourd offrait aux malfaiteurs, avec ses forêts et ses vastes landes inhabitées, des facilités pour commettre leurs crimes et disparaître ensuite, échappant à toute poursuite en passant la frontière. De là pour les habitants un manque continuel de sécurité que le représentant du roi était incapable de juguler. Ils éprouvèrent donc la nécessité de s'armer et de s'unir pour se garder eux et leurs biens. Ils imitèrent les Hermandades que le peuple avait organisées en Navarre, en Guipuzcoa et en Biscaye pour remédier à la violence créée dans le pays par l'affrontement des clans et des lignages ${ }^{17}$. Ils créerent une Armandat, soit une association armée, une "communio", qu'ils formèrent entre eux pour assister le bailli, faire eux-mêmes la police dans leur pays, se défendre mutuellement les uns les autres et même assurer la répression des crimes par la poursuite et l'arrestation des malfaiteurs.

Pour institutionnaliser leur association, ils se donnèrent des statuts qui furent approuvés en 1396 par Jean de Gand, duc d'Aquitaine, pour une durée de 4 ans. Ce délai écoulé, ils demandèrent leur renouvellement à son fils, le roi d'Angleterre Henri IV Lancastre, qui avait détrôné son cousin Richard II en 1399. Ce dernier confirma les statuts de l'Armandat du pays de Labourd, à Westminster, le 23 mars 1400, pour une durée de 20 ans.

Cette libéralité du roi duc s'explique aisément. En effet, l'Armandat permettait à son agent dans le pays d'avoir à sa disposition une force armée toujours prête à le suivre et l'aider à réprimer les désordres qu'elle lui signalait. De plus, le seigneurroi voyait là un moyen de maintenir en respect la noblesse locale et d'éviter les usurpations de seigneurs voisins.

Ce sont ces statuts, confirmés par les "lettres-patentes d'Henri IV, roi d'Angleterre et duc d'Aquitaine, approuvant les règlements et statuts faits par les habitants du Labourd, pour la poursuite des malfaiteurs ${ }^{18}$ ", ainsi que les règlements additionnels intervenus trois ans plus tard, qui font l'objet de notre étude. 
Elaborés par les "Prudhommes du Pays de Labourd", ils affichent pour but un meilleur gouvernement du pays.

Ces "prudhommes", encore appelés "hommes liges" ou encore "bonnes gens" étaient probablement les représentants des paroisses qui, élus chaque année démocratiquement, selon l'usage en Pays basque, par les maîtres de maison réunis en assemblée capitulaire à l'issue de la messe dominicale, représentaient l'ensemble des habitants du pays.

Il est d'ailleurs précisé dans ces statuts que le bailli ${ }^{19}$, qui représentait le roi en Labourd, doit réunir, trois fois par an, en sa Cour, deux bons prudhommes de chaque paroisse, élus par leurs paroissiens. Après avoir prêté serment, ces prudhommes doivent veiller à l'application des règlements de l'Armandat. Il est probable que cette Cour soit à l'origine des réunions du Bilçar, Assemblée générale de la province ${ }^{20}$, dont nous avons des procès-verbaux à partir du XVI' siècle seulement et qui, présidé par le bailli, réunissait les délégués des paroisses labourdines, pourvus d'un mandat impératif.

Après avoir reconnu la souveraineté du roi d'Angleterre et l'avoir assuré de leur fidélité, les "bonnes gens des paroisses" exigent du bailli, à son entrée en fonction dans le pays, un serment, prêté devant eux, de les bien gouverner, "comme il doit le faire", sous-entendu en respectant leurs us et coutumes. Après quoi, seulement, les délégués des paroisses lui prêteront à leur tour serment d'obéissance. On retrouve là ce pacte typiquement basque, entre les habitants et leur souverain. Leur fidélité est subordonnée au respect de leurs libertés, leurs "fueros". Ils se prémunissent là contre tout rique d'arbitraire. Il est même précisé que si le bailli refuse ce serment préalable, les Labourdins pourront refuser le leur, quitte à recourir au roi ou à l'un de ses officiers en Aquitaine.

Ces mêmes gens se sont unis entre eux par un serment réciprogue à la vie, à la mort, se jurant de s entraider contre toute personne qui voudrait leur nuire et porter atteinte à leur personne ou à leurs biens. Ce serment rappelle la conjuratio qui unissait les bourgeois des communes du nord et de l'est de la France, pays de forte féodalité, non seulement pour établir la paix dans la ville, mais aussi pour lutter contre leur seigneur et s'émanciper de sa tutelle contraignante. Une telle communio était exceptionnelle en milieu rural. Au lieu de recourir à la protection seigneuriale, les Basques, avec leur sens inné de la liberté et de la solidarité, s'unissaient pour se défendre eux-mêmes.

Les dites gens se sont notamment promis et jurés entre eux de réprimer tout vol, pillage ou autre méfait commis sur leur terre ou" autre part", par l'un des leurs, gentilhomme ou pas. Il est manifeste ici que leur objectif n'est pas seulement d'assurer la police dans leur pays, mais aussi de contenir les entreprises des nobles et des seigneurs indigènes, en Labourd ou hors de ses frontières, et de réparer les dommages qu'ils pourraient causer.

Au cas où un méfait serait commis, ils doivent tous s'assembler, avec ou sans le bailli, pour poursuivre le malfaiteur, se saisir de sa personne et le juger "à leur Cour", que le bailli ou son lieutenant y soit ou n'y soit pas. Les Labourdins exerçaient donc la Basse-justice sur leur terre à l'encontre de tout habitant du pays fauteur de trouble, quelle que fût sa condition, gentilhomme ou pas. La justice était donc encore populaire au $\mathrm{XV}^{\circ}$ siècle en Pays basque. 
Le tribunal de l'Armandat devait être cette Cour, ancêtre du Bilçar ${ }^{21}$, composée de deux prudhommes de chaque paroisse que le bailli devait périodiquement convoquer au siège du bailliage, mais qui pouvait aussi éventuellement se réunir en l'absence du bailli ou de son lieutenant, afin de donner satisfaction aux victimes d'actes dommageables en faisant donner par le délinquant "à la partie endommagée ou insultée amende pour le dommage et réparation pour l'insulte, à la connaissance du bailli et des dites bonnes gens". Comme dans les sociétés primitives donc, en l'absence d'autorité publique, amende et dommages intérêts étaient versés à la victime. Mais l'influence du droit romain se fait néanmoins sentir dans la distinction entre le dommage causé aux biens, le furtum ou le damnum injuria datum, qui donnait lieu à une amende, et l'insulte qui correspondait à l' injuria du Droit romain et qui donnait lieu à la réparation civile, soit une indemnisation comportant une idée de satisfaction, de vengeance personnelle, en cas d'atteinte à la personne ou à son honneur. Le montant de cette réparation est laissé à la discrétion de l'Armandat et du bailli, s'il est présent ; sinon, c'est l'Armandat qui la fixe.

Il est prévu que si le malfaiteur se réfugie dans la demeure ou la maison forte d'un gentilhomme ou de quiconque dans le pays, ce dernier doit être sommé par le bailli et l'Armandat de le livrer, afin que la justice puisse être rendue, "selon le le cas que fait aura”; il semble donc qu'il s'agissait d'une amende légale, sans doute celle fixée pour le cas précis dans l'ordonnance de Richard Coeur de Lion de 1190, mais qui était versée à la victime et s'ajoutait à la réparation qui lui était due. Il était alors fréquent, au $\mathrm{XVI}$ ' siecle, en Pays basque comme ailleurs, que les plus petits seigneurs fassent de leurs châteaux des lieux de refuge, où les malfaiteurs des régions voisines trouvaient limpunité. Aussi est-il précisé dans la charte que si le donneur d'asile refuse d'obtempérer, l'Armandat et le bailli pourront prendre de force, s'il le faut, la maison où s'est réfugié le malfaiteur, afin que la justice soit rendue à l'encontre du seigneur, de ses gens et de ses biens, comme du malfaiteur.

Et si ce dernier s'enfuit hors du pays, y ayant des biens, tous ses biens seront saisis pour payer l'amende et les dommages intérêts à la victime, le reste des biens étant confisqués au profit du roi ${ }^{22}$.

Mais le domaine de la juridiction de l' Armandat restait limité aux habitants du Pays de Labourd. Aussi tout homme armé, étranger ou suspect, doit être expulsé du pays au bout de 3 ou 4 jours, à moins qu'un gentilhomme ne s'en proclame en justice le garant. S'il commet un forfait, l' Armandat avec le bailli se retournerait contre son protecteur, lequel devra alors dénoncer le malfaiteur publiquement à l'église de sa paroisse à l'occasion d'une cérémonie rassemblant tous les paroissiens, sinon c'est lui qui devra répondre du délit comme s'il l'avait lui-même commis. On perçoit bien à travers ces dispositions les préoccupations de l'Armandat qui s'efforce de limiter les incursions de seigneurs voisins.

Dans leur propre paroisse, les deux prudhommes élus chaque année doivent dénoncer au bailli tout vol ou autre forfait qui s'y commettrait et le requérir de poursuivre le délinquant afin que justice soit rendue et que la victime soit satisfaite. Et si le bailli n'a pas suffisamment de forces pour agir, les gens de toutes les paroisses du pays s'assembleront et lui prêteront main forte pour poursuivre le malfaiteur et l'arrêter afin qu’il répare le préjudice commis et qu'il soit condamné suivant le cas.

Enfin. la Haute-justice est réservée au bailli. Mais en son absence ou s'il est impuissant pour arrêter le criminel ou le délinquant, L'Armandat y suppléera. Elle pourra $s^{\circ}$ emparer de lui, sans le battre ni le blesser, et le conduire, pieds et poings liés. 
au bailli où à son lieutenant pour qu'il le juge et prononce la condarnnation prévue dans ce cas ainsi que la réparation due à la victime.

A l'aube du XVc siècle donc, en Labourd, si l'autorité publique était représentée par un bailli et un lieutenant auquel il déléguait son droit de justice, leur intervention n"était nullement indispensable pour arrêter un malfaiteur, fût-il un criminel ou un gentilhomme, ou pour forcer la maison d' un seigneur et prendre le malfaiteur et ses hôtes pour en tirer justice. A la justice populaire primitive s'était ajoutée celle du représentant du seigneur justicier, mais des traces de l'état de droit primitif subsistèrent longtemps ; encore au XVIII' siècle, les malfaiteurs étaient arrêtés par le garde du pays ou par les magistrats municipaux, maire abbé ou jurats des quartiers, élus chaque année par les maîtres de maison de leur paroisse ; ils les menaient à la prison du pays pour être jugés, exerçant la police chez eux où l'intendant ou ses agents ne pénétraient guère.

-II-

Ces lettres patentes, si elles consacraient le rôle de l'Armandat auprès du bailli, manquaient cependant de précisions en ce qui concerne les délits que l'Armandat avait pour mission de poursuivre et leurs sanctions. Une simple allusion est faite à une amende légale, à côté de la réparation civile. Il était nécessaire d'actualiser le montant de ces amendes qui avaient été fixées plus de deux siècles auparavant par Richard Cour de Lion.

Les vols et pillages demeuraient nombreux. L'insécurité régnait toujours dans le pays. Par ailleurs, beaucoup ne voulaient pas reconnaître l'autorité de l'Armandat, contestée, semble-t-il, par le bailli lui-même.

Ce sont ces deux raisons, les" fréquentes voleries qui se commettent en la terre de Labourd" et les" gens qui ne veulent pas reconnaître l'Armandat", qui motivèrent la rédaction en 1403 d" "autres statuts et règlements faits par lesdits habitans et approuvez par le même Roy.", également pour 20 années "2?.

En premier lieu, il est interdit à tout Labourdin de donner asile et de protéger un étranger, à peine de 5 écus, payables la moitié au roi et l'autre moitié à l'Armandat. Et la victime d'un vol est obligée de le dénoncer à 1 " "alcalde" ${ }^{24}$ de la paroisse du voleur, à peine de 5 écus. payables comme dessus; et si ]"alcalde ne fait pas son devoir, c’est l'Armandat qui y suppléra.

En second lieu, le bailli est tenu de prêter un serment spécial. Il doit jurer de respecter les statuts et règlements de l'Armandat et de ne faire aucun tort aux habitants du pays, membres de l'Armandat, ce qui laisse supposer qu'il ne respectait guère les premiers règlements. Et, pour remédier à ces abus, les crimes, de la compétence du Haut justicier, donc du bailli ou de son lieutenant, sont aussi envisagés. Il est ainsi précisé que l'homicide ou les blessures par imprudence ne sont pas sanctionnés. Quant aux garanties judiciaires, si importantes dans le droit pénal basque, elles ne sont pas oubliées ; tout accusé d'un crime s'il est habitant du Pays de Labourd peut rester en liberté s"i] offre caution au bailli et à la partie plaignante. Si le bailli veut passer outre, l'Armandat sera tenu de prêter main forte à l'accusé.

Suit une tarification des délits et des peines, qui reprend celle de 1190. Mais des archaïsmes. tels que le serment des cojureurs, les atteintes au lignage, une amende par os extrait du crâne en cas de blessure à la tête, la course nus à travers la ville des coupables d'adultère..., ont disparu dans les nouveaux règlements. 


\section{Les principaux délits de la compétence de l'Armandat sont énumérés}

les coups et blessures, le vol, le viol, l'adultère et les blessures causées par l'animal d'autrui.

Le coupable devra d"abord "satisfaire" la victime, expression qui comporte une idée de vengeance personnelle et rappelle la composition pécuniaire du droit primitif. De plus il devra payer une amende : 2 écus pour un soufflet, 12 écus pour une blessure volontaire suffisamment grave pour qu'elle soit sanctionnée par la loi ${ }^{*}$, c'est-àdire, probablement, la charte des malfaiteurs de Richard Coeur de Lion, sinon 4 écus seulement, le double de ce qui aura été pris dans le cas de vol de nuit avec effraction ou de jour sur le grand chemin, et 20 écus pour un viol. Ces amendes seront payées la moitié au roi et l'autre moitié à l' Armandat. Et elles ne dispenseront pas les coupables de la peine prévue par la loi, qui était la pendaison en cas de vol de grand chemin ${ }^{*}$ et la mariage forcée pour le violeur si sa victime l'accepte, sinon sa livraison "corporelle" aux parents de la victime "pour en être fait à leur volonte", soit pour exercer la vengeance personnelle ${ }^{27}$, seule trace d'archaïsme que l' on retrouve implicitement dans la charte de 1403, qui, après avoir fixé l'amende en cas de viol, ajoute" s'il peut être atteint, il sera puny selon la loy civile" ${ }^{28}$.

Il est en outre précisé que le dénonciateur d'un vol recevra un écu de la victime, disposition destinée à inciter les victimes d'un vol à le dénoncer, sans crainte de représailles.

Les coupables d'adultère, s'il est notoire, sont sévèrement punis, par la confiscation de tous leurs biens, meubles et immeubles, un tiers au profit du roi, un tiers au profit de l'Armandat et un tiers pour la personne lésée. Et ils seront expulsés du pays.

Si un animal tue ou blesse quelqu'un, son propriétaire ne sera pas poursuivi. Comme en droit primitif, c'est l'animal qui est responsable ; il sera saisi et probablement vendu ; un tiers de sa valeur sera pour le roi, un tiers pour l'Armandat et un tiers pour la victime du dommage. On retrouve l'abandon noxal du droit romain qui a subsisté dans nos campagnes jusqu' au XVIII' siècle ${ }^{29}$.

L'infanticide ne doit aucune réparation, faute de victime. Mais le coupable demeure passible de la peine prévue par la loi qui était la pendaison pour un homicide.

Une dernière précision nous indique que font partie de l'Armandat tous les hommes de 14 ans et plus, lesquels sont tenus de prêter le serment de l'Armandat, et de respecter et exécuter ses règlements.

Cette institution que nous venons d'étudier présente un caractère populaire particulièrement digne d'intérêt. Une telle égalité devant le châtiment était alors exceptionnelle, si ce n'est en droit canonique.

On retrouve dans ces statuts cette fière indépendance avec laquelle les Basques, tout en se soumettant au roi qui, lointain, ne les gênait guère, savaient se prémunir contre ses officiers présents dans le pays. En leur absence, ils ne reconnaissaient plus aucune autorité ${ }^{\text {wit. }}$.

Hommes libres, nobles ou non nobles, les Labourdins ont su conserver leurs libertés. à l'abri du régime féodal comme de l'individualisme du droit romain. S'il y eut des cas de féodalité, ils furent exceptionnels et tardifs, et les habitants parvinrent à racheter aux seigneurs locaux tous leurs droits 3 .

Ils conservèrent leurs institutions après leur annexion à la couronne de France en 1451. L'Armandat prit le nom plus français de milice. Plusieurs ordonnances royales 
mentionnent la milice du Labourd comme ayant existé de temps immémorial. Cette force armée était nécessaire aux rois de France pour surveiller la frontière pendant les troubles qui régnèrent en Navarre aux $\mathrm{XV}^{*}$ et $\mathrm{XVI}^{*}$ siecles et pendant la guerre avec l'Espagne. Mais elle était désormais séparée du Bilçar et n'avait plus aucun rôle en matière judiciaire. Elle subsista, vestige du passé, composée de mille hommes, sous le commandement du bailli. Mais alors que dans les autres provinces du royaume de France, les milices locales furent absorbées, sous le nom de régiments provinciaux, dans l' armée royale permanente créée par Charles VII en 1439, la milice de Labourd, fut conservée jusqu'à la Révolution de 1789, à l'état de corps distinct et indépendant, ne relevant que du Bilçar, l'assemblée populaire du pays, d'où les nobles et les clercs étaient exclus.

Les privilèges des provinces et des communautés d'habitants ayant été emportés par la grande vague d'abolition des privileges, la nuit du 4 août 1789 , la milice du pays de Labourd fut expressément supprimée par un décret de l'Assemblée constituante du 4 mars 1791 .

Maïté LAFOURCADE

Professeur d'Histoire du droit à l'Université de Pau et des Payss de l'Adour

Faculté pluridisciplinaire de Bạonne-Anglet-Biarritz 


\section{NOTES}

1. CI. Jcan de JAURGAIN, La Vasconie. Elude historique el critique sur les origines du rovaume de Nwarte, du duché de Gascogne. des comtés de Comminges. d'Aragon. de Foix. de Bigorre, d'Alava et de Biscave, de la viconté de Béarn et des grands fiefs du duché de Gascogne. Pau, tome I, 1898, p. 207. Généalogie des vicomtes de Labourd : tome II, 1902, pp. 233-250 - Justo PEREZ DE UR BEL, Sancíno el Mavor de Navarra. Madrid, 1950, pp. 92-100.

2. Le royaume de Navarte incorporait alors la plus grande partie de l' Alava, la Biscaye et le Guipuzcoa. territoire peuplé de Vascons et qui constitue à l'hcurc actuelle la communauté autonome d'Euskadi

En ce qui conceme les liens entre la vicomte de Labourd et le royaume de Navarre, voir : Pierre YTURBIDE. "L'ancien "Armandat" du pays de Labourd", in : Revue internationale d'Etudes basques. 1907. pp. 469 ct 470 .

3. AA 11, p. 8.

4. Cette charte a ćte publiéc par Jules Balasque, Eludes historiques sur la ville de Bayonne, tome I, Bayonne, 1862, pp. 419-425.

Le dictionnaire de LESPY traduit le mot "caver". que l'on retrouve dans tout le sud-ouest, par chevalicr $\mathrm{ct} \mathrm{plusicurs} \mathrm{autcurs} \mathrm{ont} \mathrm{suivi} \mathrm{cette} \mathrm{interprétation} \mathrm{qui} \mathrm{semble} \mathrm{crronce.} \mathrm{Jean-Baptiste} \mathrm{ORPUSTAN} \mathrm{pense}$ que ce mot. d'apres son étymologic. "doit désigner un rang de noblesse supérieur..., une sorte de cher" : "Les infançons ou la noblesse ruraic dans la Basse-Navarre médiévalc : nature, fonctions, terminologic", in : Instiluciones. economia y socicdad (siglos VIII-XV), Il congreso mundial vasco. 1988. p. 260. Cclte opinion cst corroboréc par l'articlc 4 du titre I de la coutume de Labourd de 1514 : "Les seigncurs cavyers qui ont juridiction basse cntre leurs fivatiers, ne peuvent au pays de la Boun excrcer aucune juridiction contre aucuns estrangicrs. sinon seullement entre leursdictz fivatiers et cn causes civillez tant seullement". Les seigncurs cavers étaient titulaires de la justice foncière sur leurs tenanciers.

6 On n'a plus d'actes du dernier vicome, Guillaume Raymond IV de Saulı à partir de 1193. D' après Picrtc HARISTOY, Guillaume Raymond IV de Sault aurait vendu tous scs droits sur la terre de Labourd au roi d'Angleterrc en 1193, moyennant une somme de 3306 florins d'or: Recherches historiques sur le Pars basque. Bayonne, tome 1. 1883.p. 179 ; mais cet auteur ne cite pas ses sources et le florin n'a été frappé a Florence qu'à partir de 1252. Dans un factum rédigé en 1684 par Jean Duhulgo, syndic du Labourd, dans un procès contre lc fermier du domainc en Aquitaine, on peut lire que c'est en 1106 que "Guillaume, vicomtc de Labourd. du conscntement du duc de Guyenne vendit aux habilants tous ses droits sur la terre labourdinc“ : A.M. Bayonne DD 20. I ter : mais un factum, pièce unilatćrale de procédure. n'est pas unc prcuvc irrefragable. L'incertude règne donc sur la fin de la vicomté de Labourd.

7. Cf. Eugènc GOYHENECHE, Le Pavs basque: Soule-Labourd-Basse-Navarre, Pau, 1979. p. 123.

8. "Enquête orúonncec par Edouard II. pour rechercher et déterminet les droits du roi sur la terrę de Labiurd-1311" : A.M. Bayonne DD20.1.

9. "Toutc la terre de Labourd est tenuc immédiatement de notrc seigncur le roi d'Angleterrc duc d Aquitaine par les nobles ct habitants de la terre susdile".

10. "...Les dites gens sont tenus de suivre le seigncur roi avec des armes et rendre les services d'ost ct de chevauchçc a lcurs frais jusqu' au port de Caulas et au-dela aux frais du roi là ou il lui plaira dans le duché d'Aquitaine pendant un certain temps. c'cst-à-dire quarante jours". Un scul témoin. Martin de Hirigoyen. sur les ncuf interroges dit que ce sont les nobles qui doivent les services vassaliques.

11. Pcut-être ces hornmes liges, hommes du roi. hommes de Cour, prêtaient-ils un scrment particulier au roi. personncl et non collectif. ct devaient-ils se rendre a la Cour du roi ou de ses officiers, bayle ou sćnechal. lorsqu ils y étaient appcles pour rendre le service de conseil ou de justice.

12. La monnaic frappée par le vicomte de Bearn à Morlaas avait cours dans le sud-ouest et une grande partic du midi de la France.

13. “... Guillclmus Amaldi de Saut a vigenti annis citra octo hospitia. item dominus de Paganduria a vigenti annis citra decem hospitia in heremis dicti domini regis popularunt sine licentia dicti domini regis et authoritatc propria, et ipsas populationes et servitia exinde debita ocupant et ocupaverunt. ct omnimkdam bassam jurisdictioncm in praejudicium dicti domini regis."

14. Cf. Maité LAFOURCADE. Mariages en Labourd sou. l'Ancien Régime, Bilbao. 1989, p. 24, note 25

15. "Invenaire el description des privilèges. réglemens. impositions, surcharges. autres avantures el litres qui regardent le général et habirans du Pais de Labourt. après toute les découveries qui on en a put faire. fait en l annéc 1713. La présente impression a été faite di la diligence de Me Jean de Planthion, nndic général dudit Pais de Labourl en la présente année". Bayonne. 1713, p. 3. 
16. A.M. Bayonne AA11, p. 312.

17. Les Hermandades sont signalées en Navarre dès le règne de Sanche le Fort. En 1204, un traité de police $\mathrm{cl}$ de sauvegarde réciproque unissait les habitants des frontières de Navarre et d'Aragon. En 1258. une Hermandad fut établie en Basse-Navarre. En 1277. Eustache de Beaumarchais, gouverneur de Navarre. ordonna aux habitants des campagnes de former cntre eux des associations jurées pour se défendre contre les excès des grands seigneurs et des bandes qui les accompagnaient. En 1368, Don Carlos établit une Hermandad cntre la Navarre et l'Alava... Ces confréries armées avaient toutes des statuts écrits approuvés par le roi ct jurés par tous lcurs membres : Pierre YTURBDE, "L'ancien Armandal..." ; loc. cit, pp. 471472. En Guipuzcoa et en Biscaye existaient. dès le règne d'Henri II, les Alcaldes de Hermandad qui administraient la justicc dans des cas particuliers. Les statuts de l'Hermandad de Guipuzcoa furent approuves par les juntas de Guetaria en 1397 : Antonio BERISTAIN, Maria Angeles LARREA y Rafael Maria MIEZA, Fuentes de derecho penal vasco (sigIos XI-XVI), Antropologia vasca, volume V, Bilbao. 1980. pp. 197-227.

18. Ces letures patentes ont été publiés par Thomas RYMER, Foedera, conventiones, litterae et cujuscumque generis acta publica..., 3* édit., 1745, tome III, 4' partie, p. 180 (année 1400).

19. Le représentant du roi en Labourd est appelé bayle dans la chane des malfaiteurs de $1190 \mathrm{ct}$ bailli dans celle de 1400, tiưc qu'il gardera jusqu'à la fin de l'Ancien Régimc. En Labourd. le bailli élait le subordonné du sénéchal de Guyenne.

20. Cr. au sujcı du Bilçar, Maité LAFOURCADE, "Le Bilçar du pays de Labourd au XVIII sicule". in: De la res publica a los estados modemos. journées intemationales d"Histoire du droit de Saint-Sébasticn. Bilbao. 1992, pp. 125-147.

21. Le Bilçar. dissocié du tribunal du bailliagc. après l'annexion du Labourd à la couronne de france en 1451, conserva longtemps une compétence judiciaire. En effet, Louis XIV, dans 1'arrêt du Conscil du 3 juin 1660 par lequel il réforma le Bilçar. fit défense aux habitants du Labourd "de faire aucuns staruts ou ordonnances portant emprisonnement, bannissement, peine afflictive ou peines pecuniaires ny que lesdits statuts puissent ĉtre mis à exécution"; cet arrêt ne laissait aux Labourdins que la simple police et le urroit de prononcer des amendes et saisies pour violation de ses règlements. Mais encore au XVIIr" siècle. certains délits. notamment des vols faisáent parfois l'objet d une plainte au Bilçar qui décidait de livrer lc délinquant au procurcur du roi. ce qui était le cas lorsqu'il s'agissait de bohémiens ou d'etrangers. ou de s'arranger a l'amiable sans avoir recours a la justice du roi.

22. Ces demières Jispositions scmblent copiées sur les statuls de l'ancienne Hermandad t́ablic entre la Navarre et l'Aragon en 1204.

23. Ces staluts ont été publiés par Pierre YTURBIDE, "L'ancien Armandat...", loc. cil, pp. 477 a 479 ct Le Pavir Labourd avant 1789. Bayonne. 1908, pp. 45-47.

24. Terme cspagnol qui prouve l"cmprunt fait par les redacteurs des statuts labourdins à ceux des pays voisins.

25. Si la plaic cst "de loy et challenge" : cette expression signifierait, d'apres Pierre YTURBIDE. unc réparation pecuniairc tariféc par un textc de loi : Le Pan's de labourd... op. cit. p.46, note I. Il s' agissast prom bablement de la charte des malfaiteurs qui sanctionnait les blessures avec armes.

26. Article I de la charte des malfaitcurs de 1190 : Jules BALASQUE. Erudes historiques.... op. c1t.. pp $248 \mathrm{cl} 420$.

27. Articic 12 de la charte des malfaiteurs de 1190. Jules BALASQUE, Eludes historiques... op cil.. pp 250 ct 422.

28. La loi civile s'opposait a la loi ccclesiastique. Il n'y avait pas encore de difference nctic cnure le droit civil ct le droit pénal.

29. Le proprietaire dont les récoltes avaient été cndommagées par l'animal d'autrui pouvait le pignnt-

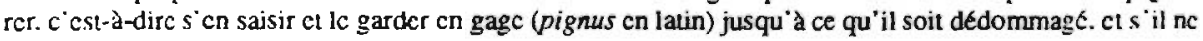
l'ćtait pas il se payait sur l'animal.

30. Cr. Yon ARRIETA ALBERDI. Vascones eta Vasconia Accursioren Magna Glossa delakoan, in : Eleria. 1. 1997, pp. 18-27.

31. En 1789, seuls les habitants de Bonloc cl de Lahonce se plaignent d'être "accablés d'enurmes redevances scigneuriales. Ce sont les seules traces de féodalité oppressivc qu" on remarque... dans ic Pays de LabourL. de tous les tems noblc. c'est-a-dire libre et allodial..." : article LXVI du "Cahier des vœeux ct des instructions des Basques François du Labourt pour lcurs députés aux Etats Généraux de la Nation". in : Pierre YTURBIDE : Cahiers des doléances de Bavonne et ditpays de Labourd pour les. Etats généraur de $1789^{\circ}$. Bayonnc. 1912. p. 49 